\title{
Combined Energy and Pressure Management in Water Distribution Systems
}

\author{
P. Skworcow ${ }^{1}$, H. AbdelMeguid ${ }^{1}$, B. Ulanicki ${ }^{1}$, P. Bounds ${ }^{1}$ and R. Patel ${ }^{2}$ \\ ${ }^{1}$ Process Control - Water Software Systems, De Montfort University, The Gateway, \\ Leicester, LE1 9BH, UK, phone: +44 116257 7070, emails: \\ \{pskworcow, habdelmeguid, bul, plmb\}@dmu.ac.uk \\ ${ }^{2}$ Yorkshire Water Services, Western House, Halifax Road, Bradford, BD6 2SZ, UK, \\ email: ridwan.patel@yorkshirewater.co.uk
}

\begin{abstract}
In this paper a method is proposed for combined energy and pressure management via integration and coordination of pump scheduling with pressure control aspects. The proposed solution involves: formulation of an optimisation problem with the cost function being the total cost of water treatment and pumps energy usage, utilisation of an hydraulic model of the network with pressure dependent leakage, and inclusion of a PRV model with the PRV set-points included as a set of decision variables. Such problem formulation led to the optimizer attempting to reduce both energy usage and leakage. The developed algorithm has been integrated into a modelling, simulation and optimisation environment called FINESSE. The case study selected is a major water supply network, being part of Yorkshire Water Services, with a total average demand of $400 \mathrm{l} / \mathrm{s}$.
\end{abstract}

\section{INTRODUCTION}

Water distribution systems, despite operational improvements introduced over the last 10-15 years, still lose a considerable amount of potable water from their networks due to leakage, whilst using a significant amount of energy for water treatment and pumping. Due to increasing energy prices, research aimed at the reduction of energy usage via the optimisation of pumps' operation (pump scheduling) has received significant interest in recent years, see e.g. (Bounds et al 2006; Ulanicki et al 2007; Ormsbee et al 2007). Minimisation of leakage, hence reduced losses of clean water and energy used for pumping and treatment, can be achieved by introducing pressure control algorithms (Ulanicki et al 2005). Whilst pump scheduling and pressure control are traditionally considered separately, if the pressure reducing valve (PRV) inlet pressure is higher than required, in many networks it could be reduced by adjusting pumping schedules in the upstream part of the network. Since many modern pumps are equipped with variable speed drives, manipulating their speed can be employed to control the pressure, thus reduce leakage and energy use.

In this paper a method is proposed for combined energy and pressure management via integration and coordination of pump scheduling with pressure control aspects. The developed algorithm has been integrated into a modelling, 
simulation and optimisation environment called FINESSE, which utilises GAMS modelling language and CONOPT non-linear programming solver. The research forms a part of the Neptune project (Neptune 2006) and aims to minimize the leakage and the energy usage whilst supplying good service to customers. In Section 2 general description of the proposed method is provided. Section 3 considers formulation of an optimal network scheduling problem. Section 4 briefly describes implementation and in Section 5 case study is presented.

\section{GENERAL METHODOLOGY DESCRIPTION}

The proposed method for combined energy and pressure management, based on formulating and solving an optimisation problem, is an extension of the pump scheduling algorithms described in (Ulanicki et al 1999; Bounds et al 2006). The main differences between these methods and the one proposed in this paper are in the network model and in the decision variables set.

The proposed method involves utilisation of an hydraulic model of the network with pressure dependent leakage and inclusion of a PRV model with the PRV set-points included as a set of decision variables. The cost function remains as in (Ulanicki et al 1999; Bounds et al 2006), i.e. represents the total cost of water treatment and pumping. Figure 1 illustrates that, with such approach, an excessive pumping contributes to a high total cost in two ways. Firstly, it leads to high energy usage. Secondly, it induces high pressure, hence increased leakage, which means that more water needs to be pumped and taken from sources. Therefore the optimizer, by minimising the total cost, attempts to reduce both energy usage and leakage.

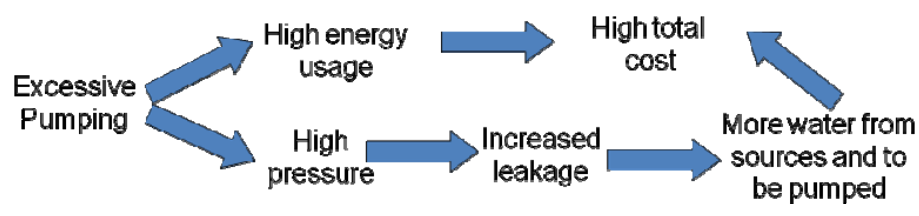

Figure 1. Illustrating how excessive pumping contributes to high total cost when network model with pressure dependent leakage is used.

In the optimisation problem considered some of the decision variables are continuous (e.g. water production, pump speed, and valve position) and some are integer (e.g. number of pumps switched on). Problems containing both continuous and integer variables are called mixed-integer problems and are hard to solve numerically. Continuous relaxation of integer variables (e.g. allowing 2.5 pumps on) enables network scheduling to be treated initially as a continuous optimisation problem solved by a non-linear programming algorithm. Subsequently, the continuous solution can be transformed into an integer solution by manual postprocessing, or by further optimisation, see (Bounds et al 2006). For example, the result " 2.5 pumps on" can be realised by a combination of 2 and 3 pumps switched over the time step. In this paper only continuous optimisation problem is considered, with the continuous solution of case studies (described in Section 5) being discretised by manual post-processing. 


\section{OPTIMAL NETWORK SCHEDULING PROBLEM}

Network scheduling calculates least-cost operational schedules for pumps, valves and treatment works for a given period of time, typically 24 hours. The decision variables are the operational schedules for control components, such as pumps, valves (including PRVs) and water works outputs. The problem has the following three elements:

1. objective function,

2. hydraulic model of the network,

3. constraints.

The scheduling problem is succinctly expressed as: minimise (pumping cost + treatment cost), subject to the network equations and operational constraints. The three elements of the problem are discussed in the following subsections. The problem is expressed in discrete-time, as in (Ulanicki et al 1999; Bounds et al 2006).

3.1. Objective function. The objective function to be minimised is the total energy cost for water treatment and pumping. Pumping cost depends on the efficiency of the pumps used and the electricity power tariff over the pumping duration. The tariff is usually a function of time with cheaper and more expensive periods. Note that other costs (such as pump switching cost) could be included in the objective function. However, the switching cost is rarely considered due to insufficient data available to formulate it within the objective function (Ormsbee and Lansey 1994). For given time step $\Delta t$, the objective function considered over a given time horizon $\left[k_{0}, k_{f}\right]$ is given by the following equation:

$$
\phi=\left(\sum_{j \in J_{p}} \sum_{k=k_{0}}^{k_{f}} \gamma_{p}^{j}(k) f_{j}\left(q^{j}(k), c^{j}(k)\right)+\sum_{j \in J_{s}} \sum_{k=k_{0}}^{k_{f}} \gamma_{s}^{j}(k) \times q_{s}^{j}(k)\right) \Delta t
$$

where $J_{p}$ is the set of indices for pump stations and $J_{s}$ is the set of indices for treatment works. The $c^{j}(k)$ vector represents the number of pumps on, denoted $u^{j}(k)$, and pump speed (for variable speed pumps) denoted $s^{j}(k)$. The function $\gamma_{p}^{j}(k)$ represents the electrical tariff. The treatment cost for each treatment works is proportional to the flow output with the unit price of $\gamma_{s}^{j}(k)$. The term $f_{j}\left(q^{j}(k), c^{j}(k)\right)$ represents the electrical power consumed by pump station $j$. The mechanical power of water is obtained by multiplying the flow $q^{j}(k)$ and the head increase $\Delta h^{j}$ across the pump station. The head increase $\Delta h^{j}$ can be expressed in terms of flow in the pump hydraulic equation, so that the cost term depends only on the pump station flow $q^{j}(k)$ and the control variable $c^{j}(k)$. From mechanical power of water, the electrical power consumed by the pump can be calculated using the pump efficiency or using pump power characteristics (Ulanicki et al 2008). 
3.2. Model of water distribution system. Each network component has a hydraulic equation. The fundamental requirement in an optimal scheduling problem is that all calculated variables satisfy the hydraulic model equations. The network equations are non-linear and play the role of equality constraints in the optimisation problem.

The network equations used in this paper to describe reservoir dynamics, components hydraulics and mass balance at reservoirs are those described in (Ulanicki et al 2007). Since leakage is assumed to be at connection nodes, the equation to describe mass balance at connection nodes is modified to include the leakage term:

$$
\mathbf{\Lambda}_{c} \mathbf{q}(k)+\mathbf{d}_{c}(k)+\mathbf{l}_{c}(k)=\mathbf{0}
$$

where $\boldsymbol{\Lambda}_{c}$ is node branch incidence matrix, $\mathbf{q}$ is vector of branch flows, $\mathbf{d}_{c}$ denotes vector of demands and $\mathbf{l}_{c}$ denotes vector of leakages calculated as:

$$
\mathbf{l}_{c}=\mathbf{p}^{\alpha} \kappa
$$

with $\mathbf{p}$ denoting vector of node pressures, $\alpha \in\langle 0.5,1.5\rangle$ denoting leakage exponent and $\kappa$ denoting vector of leakage coefficients, see (Ulanicki et al 2000). Note that $\mathbf{p}^{\alpha}$ denotes each element of vector $\mathbf{p}$ raised to the power of $\alpha$.

3.3. Constraints. In addition to equality constraints described by the hydraulic model equations, operational constraints are applied to keep the system-state within its feasible range. Practical requirements are translated from the linguistic statements into mathematical inequalities. The typical requirements of network scheduling are concerned with reservoir levels in order to prevent emptying or overflowing, and to maintain adequate storage for emergency purposes:

$$
h_{f}^{\min } \leq h_{f}(k) \leq h_{f}^{\max } \quad \text { for } \quad k \in\left[k_{0}, k_{f}\right]
$$

Similar constraints must be applied to the heads at critical connection nodes in order to maintain required pressures throughout the water network. Another constraint is on the final level of reservoirs, such that the final level is not smaller than the initial level. The control variables such as the number of pumps switched on, pump speeds or valve positions, are also constrained by lower and upper constraints determined by the features of the control components.

\section{IMPLEMENTATION}

Developed energy and pressure management scheduler was integrated into a modelling environment, called FINESSE. The scheduler, as with all tools in FINESSE, is general purpose in that it takes any data model of a network, simulates the network to initialise its decision variables for the network scheduler, and if the model is feasible it calculates the optimal schedules. Using model of a network FINESSE automatically generates optimal network scheduling problem written in a 
mathematical modelling language called GAMS (Brooke et al. 1998), which calls up a non-linear programming solver called CONOPT (Drud 1985) to calculate a continuous optimisation solution. CONOPT is a non-linear programming solver, which uses a generalised reduced gradient algorithm (Drud 1985). An optimal solution is fed back from CONOPT into FINESSE for analysis and/or further processing. For further details on using GAMS and CONOPT for optimal network scheduling see (Ulanicki et al 1999).

\section{CASE STUDY}

5.1. Network description. The case study selected is a major water supply network, being part of Yorkshire Water Services (YWS). The network is fed by two major sources and has two major exports. The network consists of 2074 nodes, 2212 pipes, 4 reservoirs, 12 pumps and 56 valves (including one PRV) with a total average demand of $400 \mathrm{l} / \mathrm{s}$. However, only 8 out of 12 pumps were actually used for scheduling, since other 4 pumps are in standby mode and should not be used unless in an emergency situation such as failure of other pumps. It was also found that only 8 out of 56 valves could be scheduled, while others are to remain fully closed. Schematic of the case study network is illustrated in Figure 2. Description of current operation of the network follows.

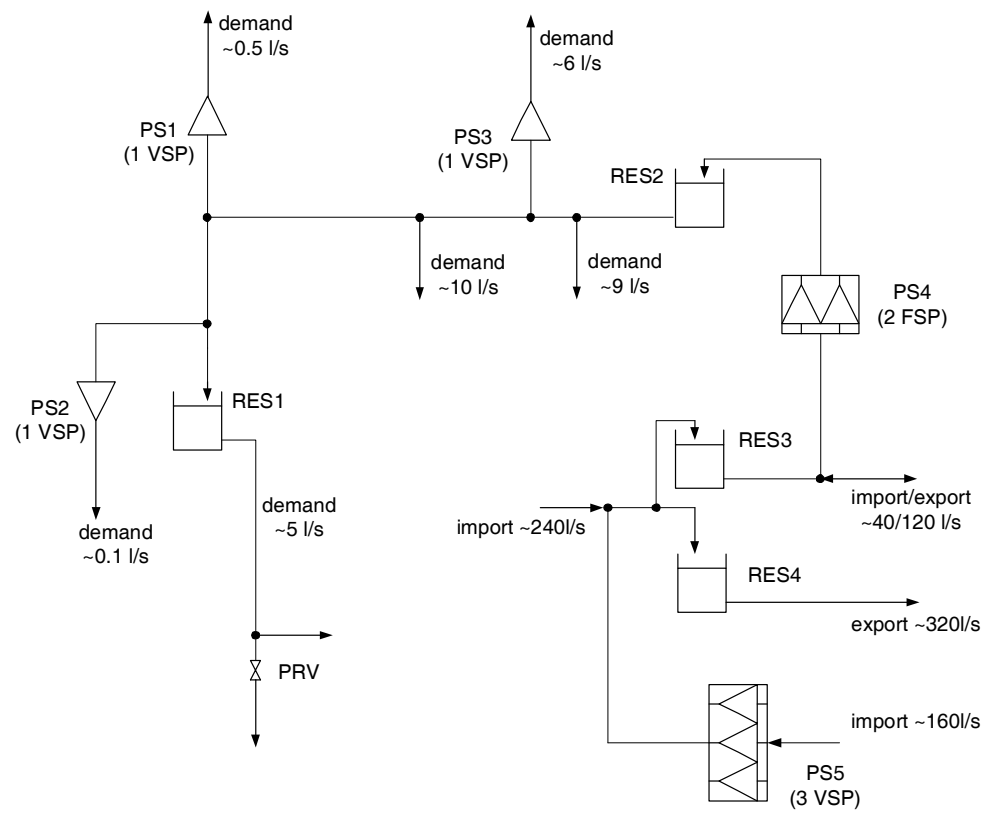

Figure 2. Illustrating schematic of the case study network. Abbreviations denote: PS - pumping station, FSP - fixed speed pump, VSP - variable speed pump, RES - reservoir. Demands and average import/exports are also depicted.

PS1, PS2 and PS3 consist of small pumps that operate to maintain given outlet pressure. Their speed, therefore, changes together with demand. PS4 is 
controlled by water level in reservoir RES2 (on/off control with $20 \mathrm{~cm}$ water level margin); typically only one pump is used. Due to tight margin $(20 \mathrm{~cm})$ the pump in PS4 is switched on/off frequently, every 7 to 30 minutes. PS5 consists of three large pumps with variable speed drives. They operate to number of preset flow bands; typically only one or two pumps are on. Opening of the top-feed valve for RES1 is controlled by water level in RES1. The top-feed valves for RES2-5 are fully open.

Information about the electrical tariffs in the considered water network was not available at the time this work was carried out. For this reason the tariffs were assumed to represent a typical scheme of cheap at night and expensive during day. Assumed tariffs were: $0.1 £ / \mathrm{kWh}$ between $22.00-07.00$ and $0.2 £ / \mathrm{kWh}$ between $07.00-22.00$.

5.2. Modelling and simplification. A model of the network was provided in Aquis format. Structure of nodes and pipes was automatically converted into EPANET format and subsequently imported into FINESSE. Other network element, i.e. reservoirs, pumps and valves, were added manually to the FINESSE model. Pumps, valves and reservoirs parameters were described in FINESSE using data from Aquis model and additional information provided by YWS.

Once the FINESSE model was completed, it was simplified using FINESSE model reduction module (Ulanicki et al 1996) to reduce the size of the optimisation problem. In the simplified model all control elements remained unchanged, but the number of pipes and nodes was reduced to 45 and 43, respectively. Both full and simplified FINESSE models were simulated and compared, with respect to pump flow and reservoir trajectories, against the reference Aquis model. It was found that in Aquis model, which allows variable simulation step, the pump in PS4 was switching at intervals as small as 7 minutes. To represent such irregular switching and model similar operation of the PS4 pump in FINESSE, where minimal time step is 15 minutes, it was assumed that e.g. 0.5 pump is on during a single time step. Both FINESSE models showed satisfactory agreement, see reservoir trajectories illustrated in Figure 3. Since RES3 and RES4 are directly connected, it is considered sufficient to compare average levels for these two reservoirs. Note that for the purpose of comparison of FINESSE models with Aquis model local control rules, such as control of RES1 top-feed valve, were kept. Subsequently, these local rules were removed to enable scheduling of all control elements.

Only limited information about leakage in the considered network was available at the time this work was carried out. For this reason the network optimiser was run for several scenarios, assuming different leakages levels. According to YWS there is a considerable leakage on the connection between PS5 and reservoirs RES3 and RES4, due to significant distance and elevation difference which require high pressure at PS5 outlet. Therefore, in the scenarios described in next subsections the leakage was assumed to be on this connection. 

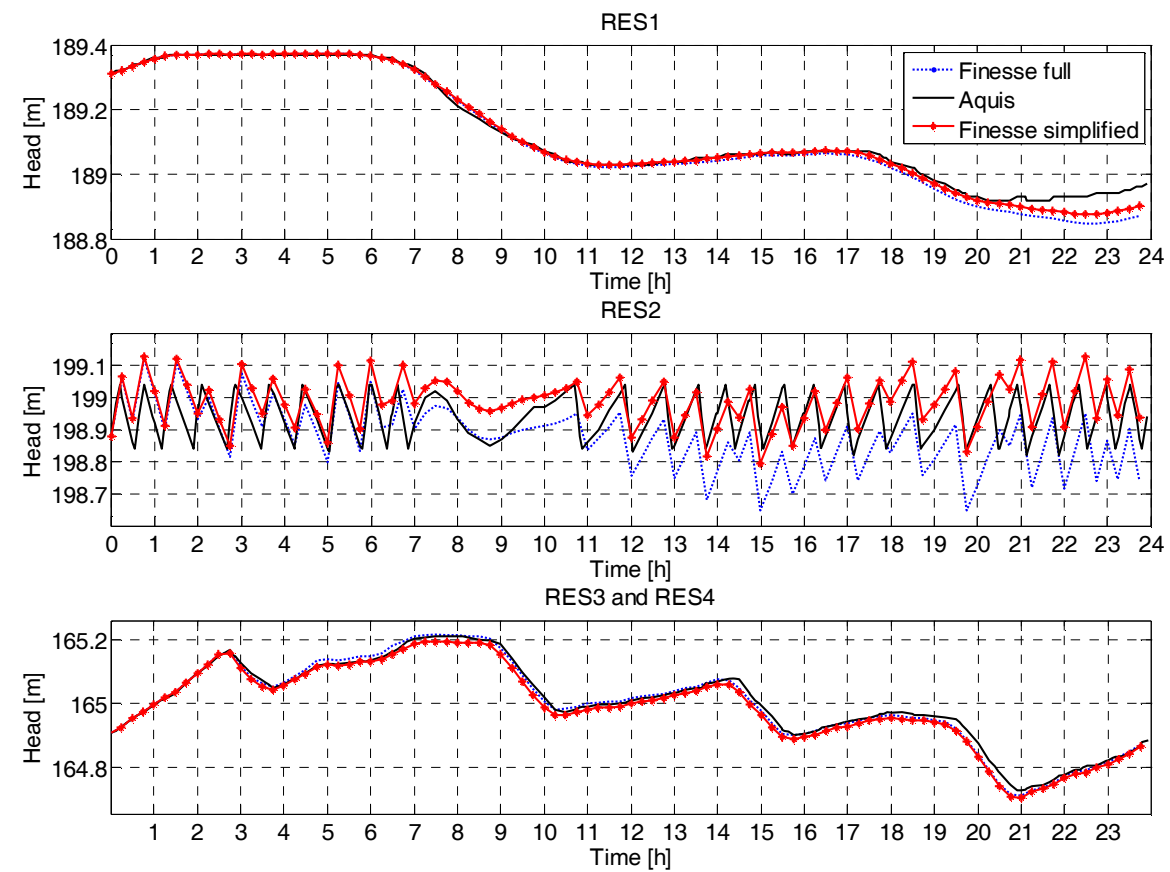

Figure 3. Illustrating reservoir level trajectories for full FINESSE model, simplified FINESSE model and reference Aquis model.

5.3. Network scheduling: no leakage. First, a case with no pressure-dependent leakage was considered, i.e. $\kappa$ in Equation (3) was zero for all nodes. Time step $\Delta t=1$ hour was chosen. The optimisation ran for 2 minutes on a Pentium $43 \mathrm{GHz}$ PC. Obtained continuous solution was transformed into an integer solution by manual post-processing. Pump schedules for PS4 and PS5 are illustrated in Figure 4 and Figure 5, respectively. Daily cost of electrical energy was as follows: $£ 799$ for current network operation, $£ 491$ for optimised continuous solution and $£ 494$ for integer solution.

5.4. Network scheduling: with leakage term. Three scenarios were considered for different leakage levels. Parameter $K$ in Equation (3) was chosen such that the leakage at a node close to the outlet of PS5 was approximately $10 \%, 20 \%$ or $30 \%$ of the flow for scenarios 1, 2 and 3, respectively. Leakage was assumed to be zero at other nodes. Only continuous solution was considered for simplicity. Obtained pump schedules for PS5 for different scenarios are illustrated in Figure 6. Daily cost of electrical energy was $£ 534, £ 547$ and $£ 562$ for scenarios 1,2 and 3 , respectively. 

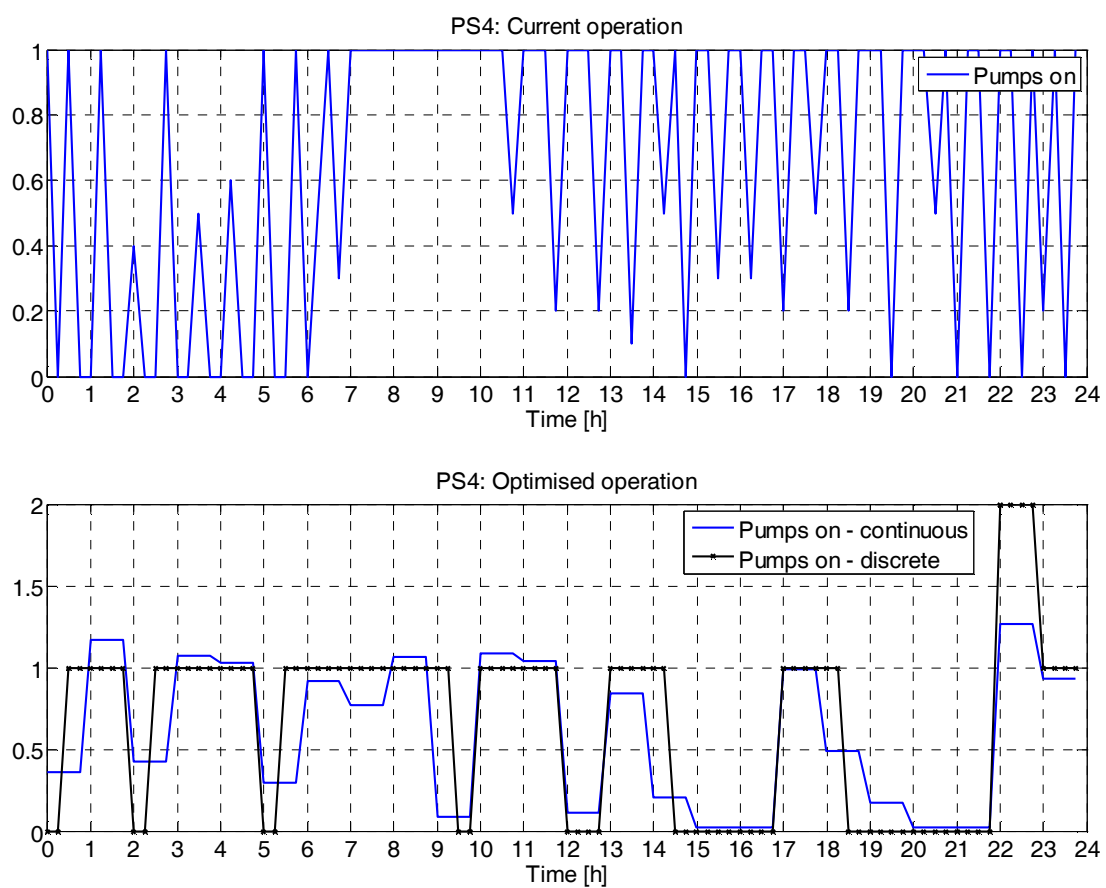

Figure 4. Comparison of current and optimised network operation for PS4.
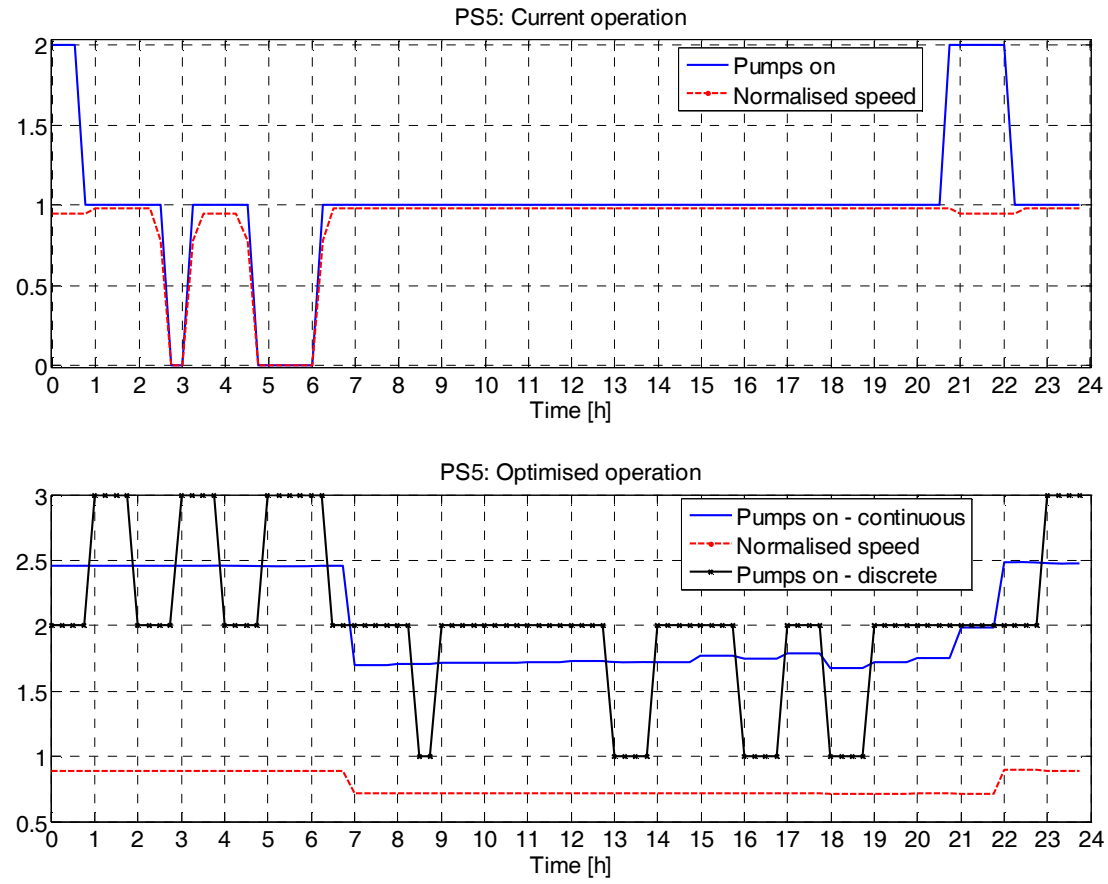

Figure 5. Comparison of current and optimised network operation for PS5. 

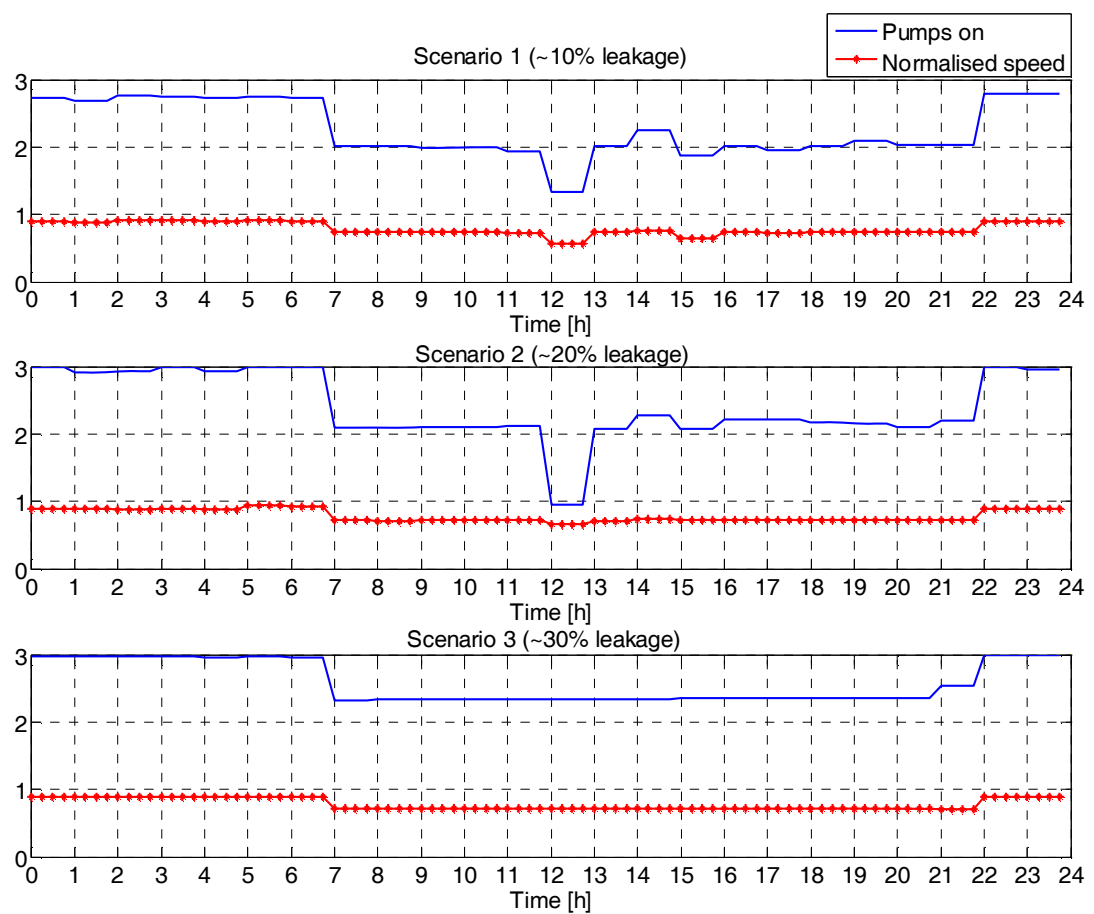

Figure 6. Comparison of PS5 pump schedules for three different leakage levels.

5.5. Discussion. It can be observed in Figure 4 that due to operational constraints not much savings can be achieved for PS4, since current and optimal schedules are similar, i.e. both exhibit switching 0-1 pumps on throughout the $24 \mathrm{~h}$ period. However, recall from Section 5.1 that PS4 switches frequently due to tight margin $(20 \mathrm{~cm})$ for reservoir level controlling the pump operation. Optimal schedules resulted in less frequent switching which is beneficial for pump durability.

In Figure 5 it can be observed that optimal schedules for PS5 cause an intensive pumping during the cheap tariff period to fill RES3 and RES4, which subsequently supply water during the expensive tariff period. The operational speed of PS5 pumps is lower, compared to current operation, during the expensive tariff period, which also contributes to reduced cost. The total cost was reduced by $38 \%$. It should, however, be noticed that these results were obtained for an assumed electrical tariff, which could significantly differ from the actual tariff, and did not take into account standing charge and other costs.

It was found that increased leakage coefficient, not surprisingly, led to increased cost, since harder pumping is required due to increased pump flow. Nevertheless, the pump schedules are similar to the 'no leakage' case, with intensive pumping during the cheap tariff period; compare Figures 5 and 6. It was observed that, despite indirect penalisation of high pressure in the cost function (see Figure 1), increased leakage coefficient did not result in lower PS5 outlet pressure. Analysis of CONOPT logs revealed that this was a result of hydraulic equation constraints: PS5 outlet pressure cannot be decreased, due to significant distance and elevation difference between PS5 and RES3. 


\section{CONCLUSIONS}

In this paper a method was proposed for combined energy and pressure management via integration and coordination of pump scheduling with pressure control aspects. The proposed solution is an extension of the pump scheduling method based on formulation and solving of an optimisation problem. Proposed method utilises an hydraulic model of the network with pressure dependent leakage and takes into account PRV model with the PRV set-points included as a set of decision variables. Such formulation leads to the optimiser attempting to reduce both energy usage and leakage. The developed algorithm has been integrated into a modelling, simulation and optimisation environment called FINESSE, which utilises GAMS modelling language and CONOPT non-linear programming solver.

The method was applied to optimise the operation of a major water supply network, being part of Yorkshire Water Services. A network model provided in Aquis format was converted to Epanet format, imported to Finesse, simplified and validated against original Aquis model. Optimised network schedules resulted in the total cost of electrical energy reduced by $38 \%$, albeit for an assumed electrical tariff, which could significantly differ from the actual tariff. The developed algorithm is currently further investigated using other case studies.

\section{REFERENCES}

Bounds P., Kahler J., Ulanicki B. (2006), "Efficient energy management of a largescale water supply system", Civil Engineering and Environmental Systems, Vol 23(3), pp. $209-220$

Brooke A., Kendrick D., Meeraus, A. and Raman, R. (1998) GAMS: A user's guide, GAMS Development Corporation, Washington, USA

Drud A.S. (1985), CONOPT: A GRG Code for large sparse dynamic non-linear optimisation problems, Mathematical Programming, 31, pp.153-191

Neptune project website www.neptune.ac.uk (2009)

Ormsbee L. and Lansey K. (2007), "Optimal control in water-supply pumping system", Journal of Water Resources Planning and Management, Vol. 120(2), pp. 237-252

Ulanicki B., Kahler J., See H. (2007), "Dynamic optimization approach for solving an optimal scheduling problem in water distribution systems", Journal of Water Resources Planning and Management, Vol. 133(1), pp. 23-32

Ulanicki B., Bounds P., Rance J., and Reynolds L. (2000), "Open and closed loop pressure control for leakage reduction”, Urban Water, Vol. 2(2), pp. 105-114

Ulanicki B., Kahler J., and B. Coulbeck B. (2008), Modeling the Efficiency and Power Characteristics of a Pump Group, Journal of Water Resources Planning and Management, Vol. 134(1), pp. 88-93

Ulanicki B., Zehnpfund A. and Martinez F. (1996), Simplification of water network models. In:Hydroinformatics 1996, Proceedings of the 2nd International Conference on Hydroinformatics, Muller, A. (Eds), vol 2,pp.493-500, Switzerland, 1996 